\title{
The Features of Electronic Conduction in InAs
}

\author{
E. Khutsishvili, Z. Chubinishvili, G. Kekelidze, I. Kalandadze, T. Qamushadze, \\ and M. Metskhvarishvili
}

\begin{abstract}
The electrical properties of $n$-type crystals of InAs compound, grown from stoichiometric melt by the horizontal zone melting method, have been investigated in the temperature range of 4.2 K-300 K before and after fast neutron irradiation up to high integral fluences of $2 \times 10^{18} n \cdot \mathrm{cm}^{-2}$. At a fixed temperature electrons concentration $(n)$ increases almost by one order during irradiation, and practically does not change with increasing of temperature. $n$ increases only slightly by increasing of temperature near $300 \mathrm{~K}$, both before and after irradiation. When $n_{0} \geq 4 \times 10^{18} \mathrm{~cm}^{-3}$ the change of $n$ during irradiation is negligible. Comparison of experimental data of mobility with theory shows that the privileged scattering mechanism of electrons at $300 \mathrm{~K}$ is scattering on optical phonons in InAs with $n \sim 10^{16}-10^{17} \mathrm{~cm}^{-3}$ and scattering on ions of impurity in InAs with $n \sim 10^{18}-10^{19} \mathrm{~cm}^{-3}$. The analysis shows that during irradiation point type scattering centers of donor-type structural defects with shallow levels in the forbidden zone appear. Consequently, the mobility decreases during irradiation. At $300 \mathrm{~K}$ in $n$-InAs sample with electrons concentration of $n_{0}=3 \times 10^{16} \mathrm{~cm}^{-3}$ the mobility decreases by 5 times after irradiation, which is equivalent to the formation of $1.5 \times 10^{19} \mathrm{~cm}^{-3}$ charged point scattering centers.
\end{abstract}

Index Terms - semiconductor, current carriers, radiation, point type scattering centers.

\section{INTRODUCTION}

InAs is one of the semiconductors, that is currently widely used in modern electronics, namely in high speed transistors, integrated circuits, IR photodetectors, injection lasers, nanowire, nanostructures, structures with quantum dots and etc. Therefore, InAs is an interesting material and needed component in semiconductor solid solutions. Papers dedicated to InAs research are become classical nowadays. However, the most interesting thing that distinguishes having high electrons mobility InAs from all elementary semiconductors and other III-V compounds is the different and unusual behavior of its electrical properties during irradiation [1]-[6]. Aukerman [1] first pointed out that the concentration of electrons in InAs crystals increases during irradiation. Kekelidze et al. [2], [3] showed that this phenomenon is unique features of InAs and, unlike of all other semiconductors, in InAs mainly donor-type radiation defects appear when it is exposed by any type and any dose of hard radiation.

Submitted on March 11, 2021

Published on April 06, 2021.

E. Khutsishviliis, F. Tavadze Institute of Metallurgy and Materials Science, Georgia.

(e-mail: elzakhutsishvili@ ${ }^{\circledR}$ yhoo.com)

Z. Chubinishvili, Georgian Technical University, Georgia.

(email: zurabchubinishvili@ yahoo.com).

G. Kekelidze, International Innovative Technologies, Georgia.

(e-mail: kekelidzeg ${ }^{\circledR}$ hotmail.com).
Despite much scientific attention to InAs, the nature of its amazing radiation properties is still far from being solved. Due to the great importance of this issue, the electrical properties of the InAs compound are the subject of our study.

\section{EXPERIMENT}

Experimental samples of InAs were grown from stoichiometric melt by the horizontal zone melting method. The data of electrical properties are obtained by the measurements of Hall effect and electric conductivity with compensation circuit at the direct current in the interval of 4.2 K-300 K. The obtained samples were $n$ - doped by Te. High degree of homogeneity of InAs crystals was confirmed by several methods, among which the most important are X-ray micro and microstructure analyses. The typical microstructure of experimental samples is shown in Fig. 1, which does not change during irradiation. The crystals were irradiated with fast neutrons up to fluencies of $\Phi=$ $2 \times 10^{18} \mathrm{n} / \mathrm{cm}^{2}$.

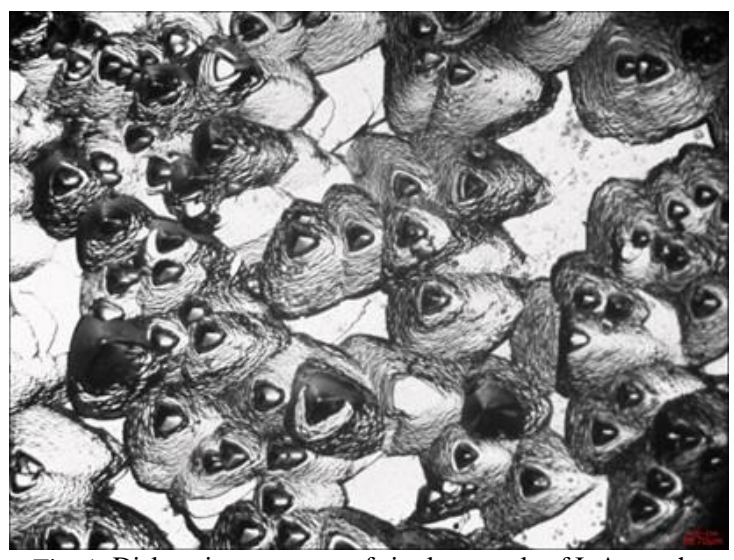

Fig. 1. Dislocation structure of single crystals of InAs on the (111) plane (x250).

\section{RESULTS AND DISCUSSION}

The mobility of current carriers is a key parameter in semiconductor devices to characterize the transfer of charged particles and the output of current. Current carriers have the ability to move quickly at high mobility values in materials. For this reason, the main task of semiconductor material science is to achieve the maximal possible value of

I. Kalandadze, Georgian technical University, Georgia (e-mail: ikalandadze@ ${ }^{\circledR}$ rambler.ru).

T. Qamushadze, F. Tavadze Institute of Metallurgy and Materials Science, Georgia.

(e-mail: t.qamushadze@gmail.com),

M. Metskhvarishvili, Georgian Technical University, Georgia. (e-mail: m.metskhvarishvili@gtu.ge). 
the current carriers mobility of the material [7]. It is especially advanced the problem of mobility of current carriers during operation of the device in an irradiated environment. The mobility itself is determined by the velocity of the current carriers, which scatter on the lattice vibrations, impurities of ions, defects in the semiconductor crystal structure. The velocity of the charge carriers is $1 / \tau$ (the inverse value of the relaxation time $\tau$ of between electrons scattering).

The values of mobility $(\mu)$ depend on the mean relaxation time $(\bar{\tau})$ between scattering as follows:

$$
\mu=\frac{q}{m^{*}} \bar{\tau}
$$

where $q$ is the elementary charge, $m^{*}$-the effective mass of the charge carriers, $\bar{\tau}$-the mean time between scattering of charge carriers.

\section{A. Unirradiated Crystals}

Fig. 2 and 3 show the mobility dependences on the current carriers concentration (at $300 \mathrm{~K}$ and $77 \mathrm{~K}$ ) and temperature respectively for the different current carriers concentration of InAs before irradiation.

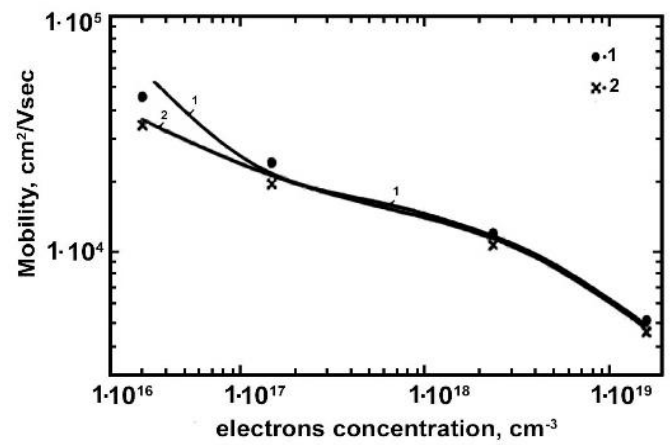

Fig. 2. Current carriers mobility dependence on the current carriers concentration before irradiation for $n$-InAs $T, K: 1-300 \mathrm{~K}, 2-77 \mathrm{~K}$.

The temperature dependencies of the mobility at the fixed current carriers concentration are determined by the different scattering mechanisms and changing of the contribution of the various components of the scattering mechanisms in total scattering when temperature is changing (Fig. 3). Existing theories allow to identify the main mechanisms of the scattering of the current carriers [8]-[12]. The total mobility depends on the mobility components, associated with the different scattering mechanisms, as the sum of the inverse values of the mobility components. Comparison of the experimental data of the mobility with the theory in InAs, where the impurity concentration is varying in the area of $10^{16}-10^{19} \mathrm{~cm}^{-3}$, shows that from all possible scattering mechanisms, electrons mobility in InAs mainly is determined by the combination of current carriers scattering on the optical phonons and ions of impurities in the temperature interval of $150 \mathrm{~K}-300 \mathrm{~K}$.

The contribution of these scattering mechanisms is different at various temperatures and electrons concentration. Calculations have shown that the privileged scattering mechanism of electrons at $300 \mathrm{~K}$ is scattering on optical phonons in InAs with $n \sim 10^{16}-10^{17} \mathrm{~cm}^{-3}$. In InAs with charge carriers of $n \sim 10^{18}-10^{19} \mathrm{~cm}^{-3}$ scattering on ions of impurity predominates. When the temperature decreases the scattering on the optical phonons decreases and the scattering on the ions of the impurities begins to dominate when $T<150 \mathrm{~K}$.

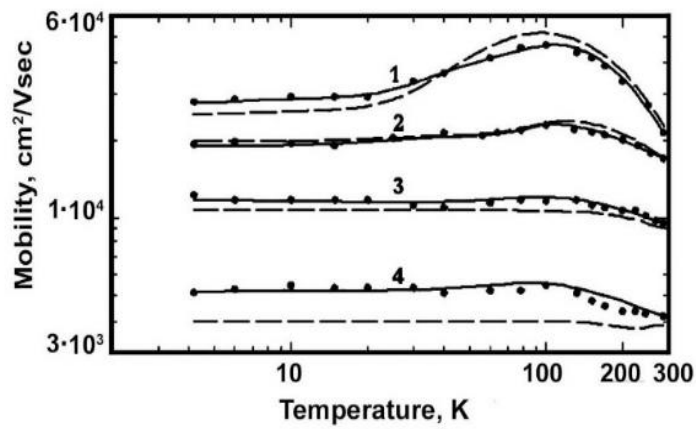

Fig. 3. The temperature dependence of mobility of unirradiated $n$-InAs, $n$, $\mathrm{cm}^{-3}$ : $1-2.9 \times 10^{16} ; 2-1.6 \times 10^{17} ; 3-2.5 \times 10^{18} ; 4-1.6 \times 10^{19}$. Curves: continuousexperimental and intermittent-theoretical data.

The existence of the maximum in the temperature dependence of the mobility is related to the changes of the contribution of the electrons scattering on the optical phonons and the ions of the impurities into the total scattering during the changes of temperature. It is seen that the observed maximum disappears at the carriers concentration of $n \sim 10^{18} \mathrm{~cm}^{-3}$.

\section{B. Crystals Irradiated with Fast Neutrons} $\left(\Phi=2 \cdot 10^{18} \mathrm{n} \cdot \mathrm{cm}^{-2}\right)$

Fig. 4 and 5 show the temperature dependences of the concentration of current carriers and mobility of InAs before and after irradiation respectively. Fig. 4 shows that at a fixed temperature $n$ is increasing significantly, almost by one order during irradiation, and practically does not change with increasing of temperature.

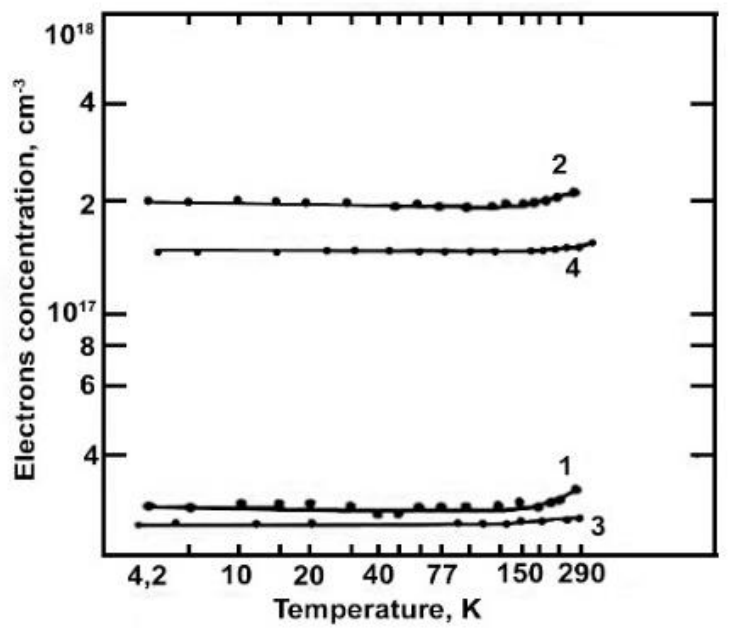

Fig. 4. Temperature dependencies of electrons concentration for InAs $\left(n_{0}=3 \times 10^{16} \mathrm{~cm}^{-3}\right)(1$ and 2$)$ and InAs doped by 10 at $\% \mathrm{P}\left(n_{0}=1.5 \cdot 10^{16} \mathrm{~cm}^{-3}\right)$ ( 3 and 4 ). 1 and 3 - before irradiation, 2 and $4-$ after irradiation by fast neutrons up to fluences of $2 \times 10^{18} n \cdot \mathrm{cm}^{-2}$. 


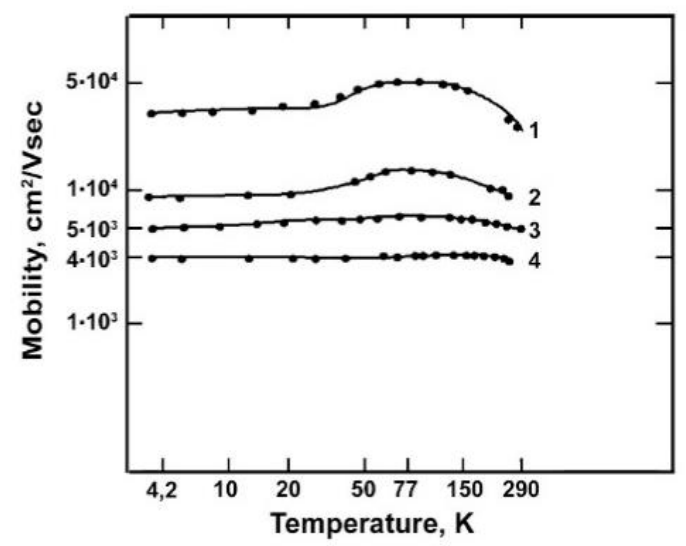

Fig. 5. Temperature dependence of current carriers mobility for InAs $\left(n_{0}=3 \times 10^{16} \mathrm{~cm}^{-3}\right)$ (curves 1 and 3$)$ and for InAs doped by 10 at $\% \mathrm{P}$ $\left(n_{0}=1.5 \times 10^{16} \mathrm{~cm}^{-3}\right)(3$ and 4$) .\left(n_{0}=1.5 \times 10^{16} \mathrm{~cm}^{-3}\right)$ (Curves 2 and 4$) ; 1$ and 2 - prior irradiation, 3 and 4 - after irradiation by fast neutrons up to fluences of $2 \times 10^{18} n \cdot \mathrm{cm}^{-2}$.

$n$ increases only slightly by increasing of temperature near $300 \mathrm{~K}$, both before and after irradiation. When $n_{0} \geq$ $4 \times 10^{18} \mathrm{~cm}^{-3}$ the changes of $n$ during irradiation is negligible. The analysis shows that the observed phenomenon is related to the occurrence of donor-type structural defects during irradiation, which form shallow levels in the forbidden zone. Consequently, the mobility (Fig. 5) decreases during irradiation. The mobility at $300 \mathrm{~K}$ in $n$-InAs sample with concentration of $n_{0}=3 \times 10^{16} \mathrm{~cm}^{-3}$ decreases by 5 times after irradiation, which is equivalent to the formation of $1.5 \times 10^{19} \mathrm{~cm}^{-3}$ charged point centers. The observed regularity in electrons concentration and mobility temperature dependences of InAs preserved in doped by 10 at\% P crystals of InAs (Fig. 4 and 5, curves 3 and 4).

\section{CONCLUSION}

Electrical properties in InAs with a concentration range of current carriers of $10^{16}-10^{19} \mathrm{~cm}^{-3}$ were studied before irradiation and during the irradiation at a temperature range of $4.2 \mathrm{~K}-300 \mathrm{~K}$. According to the temperature dependencies of the mobility of current carriers in InAs and on the base of existing theories, the main mechanisms of scattering of electrons have been revealed.

The phenomena observed during irradiation are related to the occurrence of donor-type structural defects which is equivalent to the formation of $1.5 \times 10^{19} \mathrm{~cm}^{-3}$ charged point scattering centers with shallow levels of forbidden zone. The observed regularity of temperature dependences of electrical properties of InAs preserved in doped by $10 \mathrm{at} \% \mathrm{P}$ crystals of InAs.

\section{REFERENCES}

[1] L. W. Aukerman, Electron Irradiation of Indium Arsenide, Phys. Rev. 115(5), pp.1133-1135, 1959.

[2] N. Kekelidze, G. Kekelidze. Electrical and optical properties of InP and InAs compounds and their solid solutions irradiated with fast neutrons and $\gamma$-Radiation defects in semiconductors. Institute of Physics, conference. ser.16. p. 387, Bristol, London, 1973.

[3] N. Kekelidze, G. Kekelidze. Radiation Effects in Indium Arsenide Compounds and their Solid Solutions. Radiation Effects in Semiconductors. Inst. Phys. Conf. Ser.31, Bristol, London, 1976.

[4] L.W. Aukerman, Radiation Effects, in Semiconductors and Semimetals, Edited by R. K. Willardson, Albert C. Beer, Physics of 111-V Compounds, Chapter 6, Academic Press, New York and London, 1968, Vol.4, pp.343-409.

[5] Bolshakova, I. Vasilevskii, L. Viererbl, I. Duran, N. Kovalyova, K. Kovarik, Ya.Kost, O. Makido, J. Sentkerestiova, A. Shtabalyuk, F. Shurygin. Prospects of using In-containing semiconductor materials in magnetic field sensors for thermonuclear reactor magnetic diagnostics //IEEE Transactions on Magnetics. - Volume 49, Issue 1, pp.50-53, 2013.

[6] H. Ehrenreich, Electron mobility of indium arsenide phosphide [In $\left.\left(\mathrm{As}_{\mathrm{y}} \mathrm{P}_{1-\mathrm{y}}\right)\right]$,J. Phys. Chem.Solids, 12, pp.97-104, 1959.

[7] C. M. Wolfe, N. Holonyak, Jr., and G. S. Stillman, Physical Properties of Semiconductors. Englewood Cliffs, N. J.: Prentice Hall, 1989.

[8] R. Mansfield, Impurity scattering, Proc. Phys. Soc. B.69, pp.76-82, 1956.

[9] F.J. Blatt, Theory of Mobility of Electrons in Solids, Solid State Physics,_Academic Press Inc., New York, 4, pp. 199-366, 1957.

[10] H. Brooks, Advances in Electronics and Electron Physics.7, Academic Press, New York, p.177, 1955.

[11] D. L. Rode, Electron Mobility in Direct-gap Polar Semiconductors, Phys. Rev. B2, pp.1012-1023, 1970.

[12] D. L. Rode, Electron Transport in InSb, InAs, and InP, Phys. Rev., B3, pp.3287-3299, 1971.

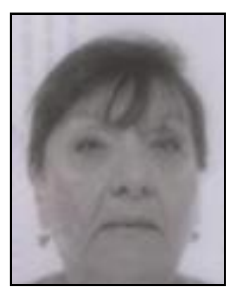

Elza Khutsishvili was born in Tbilisi, Georgia in 19.07.1941. Elza Khutsishvili has completed her PhD from Moscow Institute of Steel and Alloys in Physics and Mathematics in 1972.

She worked as the scientist of Laboratory of Semiconductor Materials, Ferdinand Tavadze Institute of Metallurgy and Materials Science from 1964 up today and the Principal research worker of Institute of Materials Research of Iv. Javakhishvili Tbilisi State University (TSU), Georgia. from 2010 up today. She has published over 140 considerable works in international journals of "Physica Status Solidi", "Solid State Communication", "Applied Optics", "Physical Review", "Physics and technique of semiconductors", including reference book "Solid Solutions in Semiconducting Systems" (Russia, Japan) and a book "Solid Solutions of Silicon-Germanium Semiconducting System" Tbilisi, Mecniereba, 1985. She has taken part with presentations in 50 more International Conferences among them in the $32^{\text {nd }}$ and $31^{\text {nd }}$ International Conferences on the Physics of Semiconductors- 2014 Austin, USA and 2012, Zurich, Switzerland accordingly.

Among published works are:

1. N. Kekelidze, E. Khutsishvili, T. Qamushadze, N. Kobulashvili, Z. Chubinishvili, G. Kekelidze. InPAs Alloys Use for Electrical Engineering in Hard-radiation Environment, European Chemical Bulletin, vol. 6(\#1), 31-35, 2021

2. R. Kobaidze, E. Kutsishvili, N. Kekelidze. Numerical computation of charge carriers optical phonon scattering mobility in III-V semiconductor compounds. Transactions of A. Razmadze Mathematical Institute, 172 (3), pp. 404-408, 2018.

3. E. Khutsishvili, B. Kvirkvelia, D. Kekelidze, V. Aliyev, D. Khomasuridze, Z. Guguchia, N. Kekelidze. Carrier mobility of InAs- and InP- rich InAs-InP solid solutions irradiated by fast neutrons. American Institute of Physics (AIP),1566(1), pp.103-104, 2013.

She has 3 patents. She is a participant of 4 international grant projects.,

Dr. E. Khutsishvili was awarded by Silver medal of Brno University, Czechoslovakia, in 1991, Consultant of 4 dissertation, editor of books.

Current and previous research interests are Semiconductor Physics, Technology, Materials Science, and Engineering.

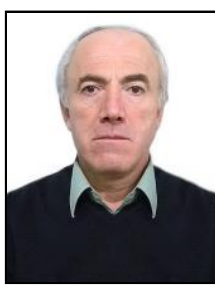

up today.

He has published over 20 considerable works in international journals. $\mathrm{He}$ has taken part with presentations in 10 more International Conferences. Among published works are:
Zurab Chubinishvili was born in Tbilisi, Georgia in 10.04.1962. Zurab Chubinishvili is PhD student at Georgian Technical University (GTU) since 2018, Tbilisi, Georgia in the field of Semiconductors and dielectrics physics. Zurab Chubinishvili's major field of study is Materials engineering and Semiconductors and dielectric of physics. He has worked as research employee at Institute of Materials Research of Iv. Javakhishvili Tbilisi State University (TSU), Georgia from 2010 
1. N. Kekelidze, E. Khutsishvili, T. Qamushadze, N. Kobulashvili, Z. Chubinishvili, G. Kekelidze. InPAs Alloys Use for Electrical Engineering in Hard-radiation Environment, European Chemical Bulletin, vol. 6(\#1), 31-35, 20211.

2. E. Khutsishvili, T. Qamushadze, N. Kobulashvili, Z. Chubinishvili, N. Kekelidze. X-ray phase analysis of impurities in silicon crystals made from metallurgical silicon with directional crystallization. European Chemical Bulletin, vol. 8(3), 85-87, 2019.

3. N. Kekelidze, B. Kvirkvelia, D. Kekelidze, E. Khutsishvili, L. Nadiradze, G. Kekelidze, T. Qamushadze, Z. Chubinishvili, I Kalandadze. Exponential Optical Absorption in Non-Irradiated and Irradiated III-V Compounds. Bulletin of the Georgian National Academy of Sciences, vol. 12, no.3, p.50-57, 2018

$\mathrm{Ph}$. D Student Z. Chubnishvili's current and previous research interests are Semiconductor Physics, Technology, Materials Science, and Engineering.

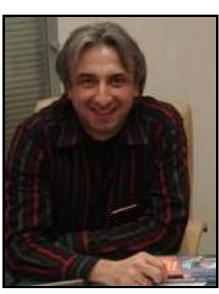

Georgy Kekelidze was born in Tbilisi, Georgia in 23.11.1964. Physist. Georgy Kekelidze Professional/MD, JD or other equivalent. Georgy Kekelidze's major field of study is Semiconductor engineering. He is Executive Director of non-profit organization "International innovative technologies". He has published over 20 considerable works. He has taken part with presentations in 20 more International Conferences.

Among published works are:

1. N. Kekelidze, E. Khutsishvili, T. Qamushadze, N. Kobulashvili, Z. Chubinishvili, G. Kekelidze. InPAs Alloys Use for Electrical Engineering in Hard-radiation Environment, European Chemical Bulletin, vol. 6(\#1), 31-35, 2021

2. R. Kobaidze, E. Khutsishvili, Z. Chubinishvili, G. Kekelidze, Computation of chemical potential and Fermi- Dirac integrals applied to study the transport phenomena of semiconductors. European Chemical Bulletin, vol. 8, \#9, 307-312, 2019

3. N. Kekelidze, B. Kvirkvelia, E. Khutsishvili, T. Qamushadze, D. Kekelidze, R. Kobaidze, Z. Chubinishvili, N. Qobulashvili, G. Kekelidze, Radiation Effects and Defects in InAs, InP Compounds and Their Solid Solutions $\operatorname{InP}_{\mathrm{x}} \mathrm{As}_{1-\mathrm{x}}$. International Journal of Physical and Mathematical Sciences. Vol:13, No:1, pp.13-18. 2019

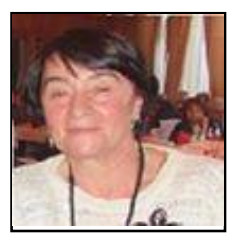

Iamze KalandaZe was born in Tbilisi, Georgia in 02.08.1947. Iamze Kalandadze has completed her $\mathrm{PhD}$ from Georgian Technical University (GTU) in 1986. Iamzse Kalandadze's major field of study is Semiconductor engineering. She is the Associate Professor of GTU, Faculty of Informatics and control Systems, Department of Engineering Physics, Georgia from 2006 up today.

She has published over 15 considerable works and 7 text books for high schools in the field of Electrical Engineering. She has taken part with presentations in 25 more International Conferences.

Among published works are:

1. N. Kekelidze, B. Kvirkvelia, D. Kekelidze, E. Khutsishvili, L. Nadiradze, G. Kekelidze, T. Qamushadze, Z. Chubinishvili, I. Kalandadze. Exponential Optical Absorption in Non-Irradiated and Irradiated III-V Compounds. Bulletin of the Georgian National Academy of Sciences, vol 12 , no.3, p.50-57, 2018

2. I. Kalandadze, T. Paghava, M. Maisuradze, M. Beridze, N. Esiava Investigation of crystals irradiated by high-energy protons through the photohall method. GTU, Transactions. no.2(492),61, 2014.

She is a participant of EU international grant project.

She has the Certificate of Iowa State University Center of Excellence in Teaching and Learning. Georgian Technical University PDC CETL. Teacher training program: Modern Methodologies in Teaching and Learning.

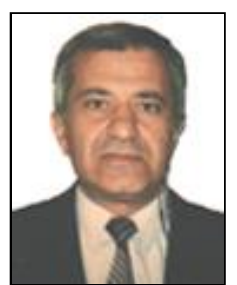

Tengiz Qamushadze was born in Tbilisi, Georgia, in 10.01.1949. Tengiz Qamushadze has completed his PhD from Iv. Javakhishvili Tbilisi State University (TSU), Georgia in Semiconductors and Dielectrics Physics, 1974. Tengiz Qamushadze's major field of study is Materials engineering and Semiconductors and dielectric of physics. He worked as senior research employee at TSU from 1974 until 2015. He is the Head of Laboratory of Semiconductor Materials, Ferdinand Tavadze Institute of Metallurgy and Materials Science of Georgia from 2015 up today.
He has published over 60 considerable works in international journals. He has taken part with presentations in 30 more International Conferences. Among published works are:

1. N. Kekelidze, E. Khutsishvili, T. Qamushadze, N. Kobulashvili, Z. Chubinishvili, G. Kekelidze. InPAs Alloys Use for Electrical Engineering in Hard-radiation Environment, European Chemical Bulletin, vol. 6(\#1), 31-35, 2021

2. E. Khutsishvili, T. Qamushadze, N. Kobulashvili, Z. Chubinishvili, N Kekelidze. X-ray phase analysis of impurities in silicon crystals made from metallurgical silicon with directional crystallization. European Chemical Bulletin, vol. 8(3), 85-87, 2019.

3. N. Kekelidze, B. Kvirkvelia, D. Kekelidze, E. Khutsishvili, L. Nadiradze, G. Kekelidze, T. Qamushadze, Z. Chubinishvili, I. Kalandadze. Exponential Optical Absorption in Non-Irradiated and Irradiated III-V Compounds. Bulletin of the Georgian National Academy of Sciences, vol. 12 , no.3, p.50-57, 2018

He has 3 Patent Inventions. Dr. T. Qamushagze's current and previous research interests are Semiconductor Physics, Technology, Materials Science, and Engineering.

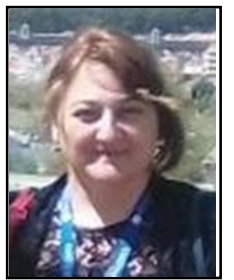

Magda Metskhvarishvili was born in Tbilisi, Georgia in 05.12.1968. Magda Metskhvarishvili has completed her PhD from Iv. Javakhishvili Tbilisi State University in 2004. Magda Metskhvarishvili's major field of study is High Temperature Materials and Semiconductors. From 1992 to 2006 she was worked in Semiconductor Materials Educational-Scientific Laboratory of Physics Faculty in Iv. Javakhishvili Tbilisi State University as a senior scientist. She is the Scientist and Invited Lecturer of Georgian Technical University (GTU) Faculty of Informatics and Control Systems, Department of Engineering Physics, Georgia from 2009 up today.

She has published over 20 considerable works in international journals and books of Journal of Superconductivity and Novel Magnetism; Journal of Materials Science: Materials in Electronics; Advanced Materials, Polymers, and Composites, AAP and CRC Press (book chapter); High Performance Functional Polymers and Composites, Apple Academic Press (book chapter); Journal of Physics: Conference Series; Journal of Low Temperature Physics; Journal of Superconductivity and Novel Magnetism; Turkish Journal of Physics. She has 3 text books for high schools in the field of General Physics. She has taken part with presentations in 40 more International Conferences among them in the Materiais2019, 14-17 April 2019, Lisbon, Portugal. Among published works are:

1. I. R. Metskhvarishvili, G. N. Dgebuadze, T. E. Lobzhanidze, B. G. Bendeliani, M. R. Metskhvarishvili, K. P. Giorgadze, V. M. Gabunia, "Influence of Dysprosium Addition on the Phase Formation and Transport Properties of Hg-1223 Superconductor", Journal of Superconductivity and Novel Magnetism, 2020, https://doi.org/10.1007/s10948-020-05634-8, Online first.

2. I. R. Metskhvarishvili, G. N. Dgebuadze, B. G. Bendeliani, M. R. Metskhvarishvili, T. E. Lobzhanidze, L. T. Gugulashvili, "Low-Field High-Harmonic Studies in Hg-1223 High-Temperature Polycrystalline Superconductor", Journal of Superconductivity and Novel Magnetism, 28 (2015) 1491

3. I. R. Metskhvarishvili, N. P. Kekelidze, M. R. Metskvarishvili. "Investigation of the Critical Current Density of $\mathrm{YBaCuO}$ hightemperature Superconductor Ceramic". Turkish Journal of Physics. 26, 2002, 299-304.

Current and previous research interests are superconductor and Semiconductor Physics. 